\title{
Supervised Model Predictive Control for discrete-time nonlinear systems with time-varying delay
}

\author{
Samah Ben Atia \\ University of Gabes \\ National Engineering school of Gabes \\ Omar Ibn Khattab street, 6029 Gabes .
} Tunisia

\author{
Anis Messaoud \\ University of Gabes \\ National Engineering school of Gabes \\ Omar Ibn Khattab street, 6029 Gabes . \\ Tunisia
}

\author{
Ridha Ben Abdennour \\ University of Gabes \\ National Engineering school of Gabes \\ Omar Ibn Khattab street, 6029 Gabes .
}

Tunisia

\begin{abstract}
The present paper deals with a decoupled multimodel predictive control based on multi-observer for the control of discrete-time nonlinear systems with time-varying delay. For each local model, a controller based on partial predictor/observer is synthesized. A switching algorithm is established to yield the adequate partial controller ensuring the closed-loop desired performances. Simulation results are given to illustrate the significance of the proposed decoupled multimodel predictive control strategy.
\end{abstract}

\section{Keywords:}

Predictive Control, Time-delay Systems, Multi-observer, Supervisor, Multimodel Approach

\section{INTRODUCTION}

Time-delay arises in many physical systems with different fields such as chemistry, biology, economic or engineering [1]. Time-delay can be generated by computational systems, actuators and sensors. In many industrial systems, the measured output is available after a time interval. This dead-time is mainly caused by the sensor technology or communication systems with low speed. It occurs also when the computing unit is located far from the controlled system.

The non consideration of the time-delay can deteriorate the performance of the control system drastically and can even lead to instability.

The Model Predictive Control (MPC) is known as an efficient control strategy for the control of industrial processes. The MPC was firstly adopted by Shell oil and knew a continual usage in several fields such as aerospace applications [2],[3],[4].

The significance of this control is due to the consideration of both performance specifications and operating constraints in the elaborated control law. The MPC uses an explicit model of the system in order to envisage its future behavior. A sequence of commands to be applied in a prediction horizon are determined. Only the first element of this sequence is actually applied to the system. This procedure is repeated at the further control intervals. So, the basic idea of the predictive control is to exploit the knowledge on the model to anticipate various situations of system operation in the future and choose the best among them according to the objectives. A state model predictive controller is proposed in [5] for MIMO nonlinear and uncertain fractional-order systems In [6], the authors proposed an improved state feedback model predictive control approach for complex systems with delayed state and input. The robustness of constrained systems with uncertainties and time-delay controlled by the MPC was studied in [7]. A robust fuzzy model predictive control joining the MPC strategy and the T-S model was discussed in [8], [9], [10], [11].

Predictive control based on nonlinear models have several limits such as the complexity of the model used in the optimisation problem. In fact, systems with nonlinear behaviors and complex structures are frequently encountered in practice [12], [13], [14]. Indeed, the handling of these systems is a difficult task in many practical situations, especially when they have highly nonlinear dynamic behaviors. Consequently, it is difficult to synthesize control laws or to implement strategies for system diagnosis for these systems with mathematical complexity. The evolution of the system around operating points is an assumption widely used in the literature [15], [16]. Therefore, a step of linearisation of the nonlinear model allows the use of techniques extensively developed with linear systems. However, in practice, the linearised model is not fully representative of the global system behavior. Thus, it is essential to find a model that provides a good characterisation of the global dynamic behavior of the nonlinear system and allows the exploitation of techniques used in the handling of linear systems. In order to meet these expectations, a new modeling technique called multimodel approach has been developed in the recent years. There are two main structures namely the coupled (Takagi-Sugeno model) and the decoupled structures. The latter structure is characterised by a further degree of freedom compared to the coupled structure. In fact, the partial models of the decoupled structure can be with different dimensions, which avoids the overparametrisation occurred with T-S model. To obtain a multimodel, different techniques have been developed in the literature. Among them, there are the linearisation [16], the convex polytopic transformation [17] and identification [18], [19].

The evolution of the system is often characterised by the evolution of its state variables. This state representation allows the synthesis of many control laws (predictive control, state feedback control...). However, the implementation of these control laws requires the knowledge of all state variables of the system which is often difficult in practice. So, it is necessary to design an observer in order to estimate the unmeasurable states [20]. Over the last decades, state estimation of nonlinear systems described by T-S 
have been widely investigated through several research works [21], [22], [23], [24], [25]. However, the state estimation of nonlinear systems represented by decoupled multimodel was recently treated in few works [26], [27].

[28] designed an observer for continuous-time decoupled multimodel with delayed output.

In fact, the accurate estimation of the state variables leads to an efficient control law. In [29], the authors proposed a nonlinear model predictive control (NMPC) based on fuzzy Kalman filter for the estimation of system states. An observer based model predictive controller considering an extended Kalman filter for discrete-time nonlinear systems was investigated in [30], [31], [32].

The main contribution of this paper is the design of a control scheme based on decoupled multimodel predictive control for a large class of discrete-time nonlinear systems with time-varying delay. On the hand, a multi-observer is investigated for the estimation of the state variables. The proposed multi-observer provides an accurate estimation required for the elaboration of an efficient multi-controller.

The present paper is organized as follows. Section two presents a multi-observer for the state estimation of decoupled multimodel with delayed output. The third section is reserved to the partial model predictive control formulation. A supervised decoupled multimodel predictive control is developed in the fourth section. Simulation example is proposed in section five to illustrate the efficiency of this control strategy. A conclusion achieves the paper.

\section{A MULTI-OBSERVER FOR DECOUPLED MULTIMODEL WITH DELAYED OUTPUT MEASUREMENTS}

\subsection{Decoupled multimodel with time-varying delay}

The multimodel approach allows the extension of linear methods to be applied to the nonlinear systems due to its easily exploitable structure. In the literature, two main types of multimodel are known. The first one is Takagi-Sugeno multimodel and the second type is known as decoupled multimodel. The last structure is retained in this work.

Consider a discrete-time nonlinear system with time-varying delay described by the following decoupled multimodel:

$$
\left\{\begin{array}{l}
x_{i}(k+1)=A_{i} x_{i}(k)+B_{i} u(k) \\
y_{m i}(k)=C_{i} x_{i}(k) \\
y_{m}(k)=\sum_{i=1}^{N m} \mu_{i}(z(k-d(k))) C_{i} x_{i}(k-d(k))
\end{array}\right.
$$

with:

$x_{i} \in \Re^{n_{i}}$ and $y_{m i} \in \Re^{p}$ are the state and the output vectors of the $i^{t h}$ partial model, respectively.

$u \in \Re^{m}$ is the input and $y_{m} \in \Re^{p}$ is the output vector.

$A_{i} \in \Re^{n_{i} \times n_{i}}, B_{i} \in \Re^{n_{i} \times m}$ and $C_{i} \in \Re^{p \times n_{i}}$ are known matrices with appropriate dimensions. $d(k)$ is a time-varying delay on output measurements. It is assumed known and it satisfies the following condition:

$$
d_{\min } \leq d(k) \leq d_{\max }
$$

with $d_{\min }$ and $d_{\max }$ are constant positive scalars denoting the lower and the upper time-delays, respectively.

$N_{m}$ is the number of partial models.

$$
\begin{gathered}
\mu_{i}(z(k-d(k)))=\frac{e^{\left(\frac{-\left(z(k-d(k))-c_{i}\right)^{2}}{\sigma_{d}^{2}}\right)}}{\sum_{i=1}^{N_{m}} e^{\left(\frac{-\left(z(k-d(k))-c_{i}\right)^{2}}{\sigma_{d}^{2}}\right)}}, \\
i=1,2, \ldots, N_{m}
\end{gathered}
$$

$c_{i}$ are the centers and $\sigma_{d}$ is the dispersion.

The $\mu_{i}(z(k-d(k)))$ are the weighting functions ensuring the transition between the partial models.

The weighting functions have the following properties:

$\sum_{i=1}^{N_{m}} \mu_{i}(z(k-d(k)))=1$,

$0 \leq \mu_{i}(z(k-d(k))) \leq 1$,

$\forall i=1, \ldots, N_{m}, \forall k$

$z(k-d(k))$ is the decision variable. This variable can be measurable such as the input, the output and the measurable states, or unmeasurable such as the unmeasurable states of the system.

Consider the equation (1). This last can be written in a compact form by defining the following vector $x(k)$ :

$x(k)=\left[x_{1}^{T}(k) \cdots x_{i}^{T}(k) \cdots x_{N_{m}}^{T}(k)\right]^{T} \in \Re^{n}$,

$n=\sum_{i=1}^{N_{m}} n_{i}$

So, the compact form of the system (1) can be written as follows [26]:

$$
\left\{\begin{array}{l}
x(k+1)=\mathbb{A} x(k)+\mathbb{B} u(k) \\
y_{m}(k)=\mathbb{C}(k-d(k)) x(k-d(k))
\end{array}\right.
$$

where $\mathbb{A} \in \Re^{n \times n}, \mathbb{B} \in \Re^{n \times m}$ and $\mathbb{C} \in \Re^{p \times n}$ are the matrices defined as follows:

$\mathbb{A}=\left[\begin{array}{ccccc}A_{1} & 0 & 0 & 0 & 0 \\ 0 & \ddots & 0 & 0 & 0 \\ 0 & 0 & A_{i} & 0 & 0 \\ 0 & 0 & 0 & \ddots & 0 \\ 0 & 0 & 0 & 0 & A_{N_{m}}\end{array}\right], \mathbb{B}=\left[\begin{array}{c}B_{1} \\ \vdots \\ B_{i} \\ \vdots \\ B_{N_{m}}\end{array}\right]$

The matrix $\mathbb{C}(k-d(k))$ can be written as follows:

$$
\mathbb{C}(k-d(k))=\sum_{i=1}^{N_{m}} \mu_{i}(z(k-d(k))) \mathbb{C}_{i}
$$

with $\mathbb{C}_{i}$ is a bloc matrix of the form:

$$
\mathbb{C}_{i}=\left[0 \ldots C_{i} \ldots 0\right]
$$

\subsection{State estimation of decoupled multimodel with time-varying delay}

The following multi-observer is considered for the state estimation of the system (1):

$$
\left\{\begin{array}{l}
\hat{x}_{i}(k+1)=A_{i} \hat{x}_{i}(k)+B_{i} u(k)+L_{i}\left(y_{d}(k)-\hat{y}_{m}(k)\right) \\
\hat{y}_{m}(k)=\sum_{i=1}^{N_{m}} \mu_{i}(z(k-d(k))) C_{i} \hat{x}_{i}(k-d(k))
\end{array}\right.
$$

where $L_{i} \in \Re^{n_{i} \times p}$ is the unknown gain associated to the $i^{t h}$ partial model and $y_{d}(k)$ is the delayed output of the system.

The multi-observer (5) is written in the compact form given by the 
following expression:

$$
\left\{\begin{array}{l}
\hat{x}(k+1)=\mathbb{A} \hat{x}(k)+\mathbb{B} u(k)+\mathbb{L}\left(y_{d}(k)-\hat{y}_{m}(k)\right) \\
\hat{y}_{m}(k)=\mathbb{C}(k-d(k)) \hat{x}(k-d(k))
\end{array}\right.
$$

with $\mathbb{L}=\left[\begin{array}{lllll}L_{1} & \cdots & L_{i} & \cdots & L_{N_{m}}\end{array}\right]^{T} \in \Re^{n}$

Thereafter, $\mathbb{C}(k-d(k))$ will be denoted by $\mathbb{C}_{d}(k)$.

The following theorem gives the convergence conditions formulated in terms of linear matrix inequalities.

Theorem Consider the system (4) and the multi-observer (6). The estimation error is asymptotically stable if there exist symmetric matrices $(P>0)$ and $(Q>0)$ and a matrix $X$ such that the following linear matrix inequalities hold:

$$
\begin{gathered}
{\left[\begin{array}{ccc}
\mathbb{A}^{T} P \mathbb{A}+\bar{d} Q-P & -\mathbb{A}^{T} X \mathbb{C}_{i} & 0 \\
\mathbb{C}_{i}^{T} X^{T} \mathbb{A} & -Q & \mathbb{C}_{i}^{T} X^{T} \\
0 & X \mathbb{C}_{i} & -P
\end{array}\right]<0} \\
i=1 \ldots N_{m}
\end{gathered}
$$

with $\quad \bar{d}=d_{\max }-d_{\min }+1$.

The observer gain is $\mathbb{L}=P^{-1} X$

Proof:The estimation error is defined as follows:

$$
\tilde{x}(k+1)=x(k+1)-\hat{x}(k+1)
$$

Consider the following Lyapunov-Krasovskii functional:

$$
V(k)=V_{1}(k)+V_{2}(k)+V_{3}(k)
$$

with

$$
\begin{gathered}
V_{1}(k)=\tilde{x}^{T}(k) P \tilde{x}(k) \\
V_{2}(k)=\sum_{i=k-d(k)}^{k-1} \tilde{x}^{T}(i) Q \tilde{x}(i) \\
V_{3}(k)=\sum_{j=-d_{\max }+2}^{-d_{\min }+1} \sum_{i=k+j-1}^{k-1} \tilde{x}^{T}(i) Q \tilde{x}(i)
\end{gathered}
$$

The forward difference $\Delta V_{1}(k)$ is obtained by the following expression:

$$
\begin{aligned}
\Delta V_{1}(k) & =V_{1}(k+1)-V_{1}(k) \\
& =\tilde{x}^{T}(k+1) P \tilde{x}(k+1)-\tilde{x}^{T}(k) P \tilde{x}(k) \\
& =\tilde{x}_{a}^{T}(k) \Gamma(k) \tilde{x}_{a}(k)
\end{aligned}
$$

where $\tilde{x}_{a}(k)=\left[\begin{array}{c}\tilde{x}(k) \\ \tilde{x}(k-d(k))\end{array}\right]$

$\Gamma(k)=\left[\begin{array}{cc}\mathbb{A}^{T} P \mathbb{A}-P & -\mathbb{A}^{T} P \mathbb{L} \mathbb{C}_{d}(k) \\ -\mathbb{C}_{d}^{T}(k) \mathbb{L}^{T} P \mathbb{A} & \mathbb{C}_{d}^{T}(k) \mathbb{L}^{T} P \mathbb{L} \mathbb{C}_{d}(k)\end{array}\right]$

The forward difference $\Delta V_{2}(k)$ is:

$$
\begin{aligned}
\Delta V_{2}(k)= & V_{2}(k+1)-V_{2}(k) \\
= & \tilde{x}^{T}(k) Q \tilde{x}(k)-\tilde{x}^{T}(k-d(k)) Q \tilde{x}(k-d(k)) \\
& +\sum_{i=k+1-d(k+1)}^{k-1} \tilde{x}^{T}(i) Q \tilde{x}(i) \\
& -\sum_{i=k+1-d(k)}^{k-1} \tilde{x}^{T}(i) Q \tilde{x}(i)
\end{aligned}
$$

It is clear that:

$$
\begin{aligned}
\sum_{i=k+1-d(k+1)}^{k-1} \tilde{x}^{T}(i) Q \tilde{x}(i) & \leq \sum_{i=k+1-d_{\max }}^{k-d_{\min }} \tilde{x}^{T}(i) Q \tilde{x}(i) \\
& +\sum_{i=k+1-d(k)}^{k-1} \tilde{x}^{T}(i) Q \tilde{x}(i)
\end{aligned}
$$

then

$$
\begin{aligned}
\Delta V_{2}(k) \leq & \tilde{x}^{T}(k) Q \tilde{x}(k)-\tilde{x}^{T}(k-d(k)) Q \tilde{x}(k-d(k)) \\
& +\sum_{i=k+1-d_{\max }}^{k-d_{\min }} \tilde{x}^{T}(i) Q \tilde{x}(i)
\end{aligned}
$$

The forward difference $\Delta V_{3}(k)$ is:

$$
\begin{aligned}
\Delta V_{3}(k)= & V_{3}(k+1)-V_{3}(k) \\
= & \tilde{x}^{T}(k)\left(d_{\max }-d_{\min }\right) Q \tilde{x}(k) \\
& -\sum_{i=k+1-d_{\max }}^{k-d_{\min }} \tilde{x}^{T}(i) Q \tilde{x}(i)
\end{aligned}
$$

Thus, the forward difference of the Lyapunov functional $V(k)$ is given by the following equation:

$$
\begin{aligned}
\Delta V(k)= & \Delta V_{1}(k)+\Delta V_{2}(k)+\Delta V_{3}(k) \\
& \leq \tilde{x}_{a}^{T}(k) \Gamma(k) \tilde{x}_{a}(k)+\bar{d} \tilde{x}^{T}(k) Q \tilde{x}(k) \\
& -\tilde{x}^{T}(k-d(k)) Q \tilde{x}(k-d(k))
\end{aligned}
$$

then

$$
\Delta V(k) \leq \tilde{x}_{a}^{T}(k) \Omega(k) \tilde{x}_{a}(k)
$$

where

$$
\begin{aligned}
& \Omega(k)=\left[\begin{array}{cc}
\mathbb{A}^{T} P \mathbb{A}-P+\bar{d} Q & -\mathbb{A}^{T} P \mathbb{L} \mathbb{C}_{d}(k) \\
-\mathbb{C}_{d}^{T}(k) \mathbb{L}^{T} P \mathbb{A} & \mathbb{C}_{d}^{T}(k) \mathbb{L}^{T} P \mathbb{L} \mathbb{C}_{d}(k)-Q
\end{array}\right] \\
& \text { and } \bar{d}=d_{\max }-d_{\min }+1
\end{aligned}
$$

The use of weighting functions properties and the application of Schur Complement lead to the following linear matrix inequalities.

$$
\begin{gathered}
{\left[\begin{array}{ccc}
\mathbb{A}^{T} P \mathbb{A}+\bar{d} Q-P & -\mathbb{A}^{T} X \mathbb{C}_{i} & 0 \\
\mathbb{C}_{i}^{T} X^{T} \mathbb{A} & -Q & \mathbb{C}_{i}^{T} X^{T} \\
0 & X \mathbb{C}_{i} & -P
\end{array}\right]<0} \\
i=1 \ldots N_{m}
\end{gathered}
$$

\section{PARTIAL MODEL PREDICTIVE CONTROL FORMULATION}

The MPC is a model-based control strategy that knew continual success in recent years. Its significance is mainly due to its efficiency to control multivariable, constrained, unstable and non-minimum phase systems. The synthesis of this control is based on the minimisation of a criterion $J_{i}$.Indeed, the optimal control is obtained by minimizing the finite horizon linear quadratic cost function:

$$
J_{i}=\frac{1}{2}\left\{\sum_{j=1}^{N_{p}}\left\|\hat{y}_{i}(k+j / k)-y_{c}(k+j)\right\|_{\nu}^{2}+\sum_{j=0}^{N_{u}-1}\left\|\Delta u_{i}(k+j)\right\|_{\eta}^{2}\right\}
$$


where :

$N_{p}$ is the prediction horizon and $N_{u}$ is the control horizon $\nu$ and $\eta$ are positive weighting factors.

$y_{c}(k+j / k)$ and $\hat{y}_{i}(k+j / k)$ are the $j$ steps reference signal and predicted partial output, respectively.

$\Delta u_{i}(k+j)$ is the future control increments $\left(\Delta u_{i}(k+j)=0 \quad \forall j \in\right.$ $\left.\left[N_{u}, N_{p}\right]\right)$.

For each partial model and based on equation (1), the corresponding estimated state predicted for $j$ steps ahead is given by the following expression:

$$
\begin{aligned}
& \left\{\begin{array}{l}
\hat{x}_{i}(k+j)=A_{i} \hat{x}_{i}(k+j-1)+B_{i} u_{i}(k+j-1) \\
\hat{y}_{i}(k+j)=C_{i} \hat{x}(k+j)
\end{array}\right. \\
& \forall j=1, \ldots, N_{p}
\end{aligned}
$$

The prediction of partial model output $\hat{y}_{i}(k+j)$ for $j$ steps ahead leads to the following expression written in a matrix form:

$$
\hat{Y}_{i}(k)=\Xi_{i} \hat{x}_{i}(k)+\Psi_{i} U_{i}(k)
$$

where

$$
\begin{aligned}
& \Xi_{i}=\left[\begin{array}{c}
C_{i} A_{i} \\
C_{i} A_{i}{ }^{2} \\
\vdots \\
C_{i} A_{i}^{N_{p}}
\end{array}\right], \Psi_{i}=\left[\begin{array}{cccc}
C_{i} B_{i} & 0 & \cdots & 0 \\
C_{i} A_{i} B_{i} & \ddots & \ddots & \vdots \\
\vdots & \ddots & \ddots & 0 \\
C_{i} A_{i}^{N_{p}-1} B_{i} & \cdots & C_{i} A_{i} B_{i} & C_{i} B_{i}
\end{array}\right] \\
& U_{i}(k)=\left[\begin{array}{llll}
u_{i}(k) & u_{i}(k+1) & \cdots & u_{i}\left(k+N_{p}-1\right)
\end{array}\right]^{T} \\
& \hat{Y}_{i}(k)=\left[\begin{array}{llll}
\hat{y}_{i}(k+1) & \hat{y}_{i}(k+2) & \cdots & \hat{y}_{i}\left(k+N_{p}\right)
\end{array}\right]^{T}
\end{aligned}
$$

The matrix form of equation (21) is given by the following expression:

$$
J_{i}=\frac{1}{2}\left[\left\|\hat{Y}_{i}(k)-Y_{c}(k)\right\|_{\vartheta}^{2}+\left\|\Delta U_{i}(k)\right\|_{\aleph}^{2}\right]
$$

where

$$
\begin{aligned}
& Y_{c}(k)=\left[\begin{array}{llll}
y_{c}(k+1) & y_{c}(k+2) & \cdots & y_{c}\left(k+N_{p}\right)
\end{array}\right]^{T} \\
& \Delta U_{i}(k)=\left[\begin{array}{llll}
\Delta u_{i}(k) & \cdots & \Delta u_{i}\left(k+N_{u}-1\right) & \overbrace{0} \cdots \quad 0
\end{array}\right]^{N_{p}-N_{u}} \\
& \vartheta=\left[\begin{array}{cccc}
\nu & 0 & \cdots & 0 \\
0 & \ddots & \ddots & \vdots \\
\vdots & \ddots & \ddots & 0 \\
0 & \cdots & 0 & \nu
\end{array}\right],
\end{aligned}
$$

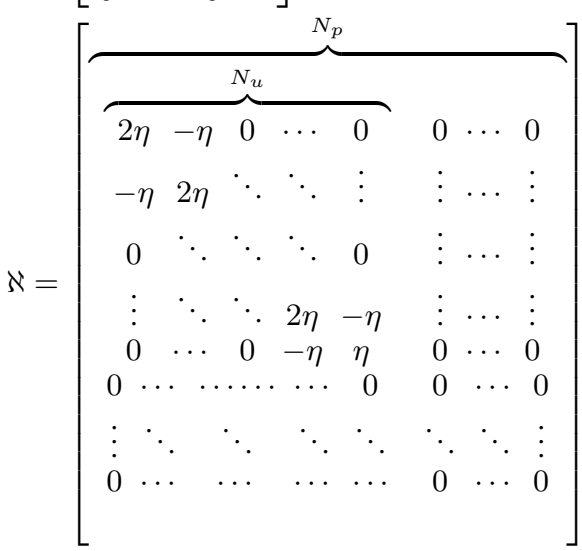

The partial criterion $J_{i}$ is given as follows:

$$
J_{i}=\frac{1}{2} U_{i}^{T}(k) \Theta_{i} U_{i}(k)+U_{i}^{T}(k) \varphi_{i}+T_{i}
$$

with

$$
\begin{aligned}
& \Theta_{i}=\Psi_{i}^{T} \vartheta \Psi_{i}+\aleph \\
& \varphi_{i}=\left[\Psi_{i}^{T} \vartheta \Xi_{i}-\Psi_{i}^{T} \vartheta\right]\left[\begin{array}{c}
\hat{x}_{i}(k) \\
Y_{c}(k)
\end{array}\right]-\left[\begin{array}{c}
\eta u_{i}(k-1) \\
0 \\
\vdots \\
0
\end{array}\right] \\
& T_{i}=\left(\Xi_{i} \hat{x}_{i}(k)-Y_{c}(k)\right)^{T} \vartheta\left(\Xi_{i} \hat{x}_{i}(k)-Y_{c}(k)\right)+\eta u_{i}^{2}(k-1) \\
& T_{i} \text { is a constant independent from } U_{i}(k) .
\end{aligned}
$$

The partial derivative of the criterion $J_{i}$ is given by the following expression:

$$
\frac{\partial J_{i}}{\partial U_{i}(k)}=\Theta_{i} U_{i}(k)+\varphi_{i}
$$

The annulment of the derivative of $J_{i}$ leads to the optimal control law:

$$
U_{i}(k)=-\Theta_{i}^{-1} \varphi_{i}
$$

\section{DECOUPLED MULTIMODEL PREDICTIVE CONTROL WITH SUPERVISION}

In practice, the state variables are often unmeasurable, so, it is necessary to synthesize an observer in order to estimate these states. In this work, a multi-observer for the state estimation of discrete-time decoupled multimodel with delayed output measurements is exploited in the elaboration of the control law. Indeed, the control scheme (figure 1)includes three main blocks; multi-observer/multi-controller, supervisor and switching system. In the first block, a multi-observer is designed in order to provide an accurate estimation of the state variables. These estimates are used thereafter to compute the multi-predictor and to elaborate the partial control laws. On the other hand, the supervisor contains a multi-predictor that describes the system behavior in the different operating regimes and a performance evaluator that has as role to evaluate a performance criterion $J c_{i}(k)$ based on the error between the system and the partial predictors outputs. Thus, the criterion having the minimal value corresponds to the suitable partial predictor and therefore a switching system selects the adequate local controller.

The following criterion is minimal for the nearest predictor output to the system output. It has the following expression [33], [34], [35]:

$$
J c_{i}(k)=\alpha \varepsilon_{i}^{2}(k)+\beta \sum_{j=1}^{k} e^{-\lambda(k-j)} \varepsilon_{i}^{2}(j), \quad i=1 \ldots N_{m}
$$

where

$\varepsilon_{i}(k)\left(\varepsilon_{i}(k)=y_{d}(k)-\hat{y}_{c i}(k)\right)$ is the error between the real and the $i^{\text {th }}$ predictor output.

$\alpha$ and $\beta$ are positive parameters denoting the weighting factors of the instantaneous and the long-term measures accuracy.

$\lambda$ is the forgetting factor denoting the memory of the index.

$N_{m}$ is the number of the partial controllers.

$\hat{y}_{c i}(k)$ is the $i^{t h}$ predictor output, it follows from the inversion of the $i^{t h}$ partial controller and it has the following expression: 


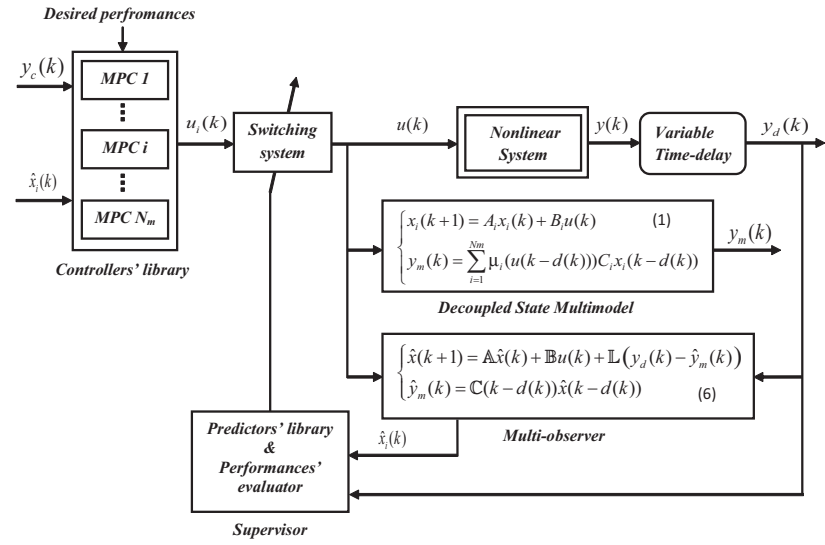

Fig. 1. Supervised decoupled multimodel predictive control based on multi-observer.

$$
\begin{array}{r}
\hat{Y}_{c i}(k)=\left(\Psi_{i}^{T} \vartheta\right)^{-1}\left(\Psi_{i}^{T} \vartheta \Psi_{i}+\aleph\right) U_{i}(k)+\Xi_{i} \hat{x}_{i}(k) \\
-\left(\Psi_{i}^{T} \vartheta\right)^{-1}\left[\begin{array}{c}
\eta u_{i}(k-1) \\
0 \\
\vdots \\
0
\end{array}\right]
\end{array}
$$

Only the first element is considered.

$$
\hat{y}_{c i}(k)=\hat{Y}_{c i}(1)
$$

\section{SIMULATION RESULTS}

The multimodel representation of a nonlinear system can be obtained by several methods such as the linearisation method, the convex polytopic transformation and the identification method. This multimodel representation reduces the identification problem of nonlinear systems to the identification of the partial models. The last method concerns the research of an optimal structure (through the choice of the decision variable and the partition of the operating space into operating zones), the estimation of the multimodel parameters and the validation of the result.

The estimation of the multimodel parameters requires the use of an optimisation method generally based on the minimisation of the error between the system and multimodel outputs. In this work, the Levenberg-Marquardt algorithm is adopted. This method ensures both the stability and the rapidity of the convergence [36].

Consider the discrete-time nonlinear system described by the following expression [37:

$$
y(k)=\frac{(y(k-1) u(k-1))}{\left(1+y^{2}(k-1)\right)}-\tan (u(k-1))
$$

The input signal $u(k) \in[-1,1]$ is the decision variable. The centers and the dispersion have the following values: $c_{1}=-0.7, \quad c_{2}=0, \quad c_{3}=0.7, \quad$ and $\quad \sigma_{d}=0.3$ The identification of the system (31) leads to the following partial models:

\section{Partial model 1:}

$A_{1}=\left[\begin{array}{cc}-0.6965 & 0.2218 \\ 0.2430 & 0.0914\end{array}\right], B_{1}=\left[\begin{array}{l}-1.1485 \\ -0.0905\end{array}\right] C_{1}=\left[\begin{array}{ll}1 & 0\end{array}\right]$.

Partial model 2:

$A_{2}=\left[\begin{array}{cc}-0.6274 & -0.3475 \\ 0.3308 & 0.1425\end{array}\right], B_{2}=\left[\begin{array}{c}-1.5599 \\ 0.1579\end{array}\right], C_{2}=\left[\begin{array}{ll}1 & 0\end{array}\right]$.

Partial model 3:

$A_{3}=\left[\begin{array}{cc}-0.2884 & -0.5253 \\ 0.2000 & 0.1818\end{array}\right], B_{3}=\left[\begin{array}{c}-2.1989 \\ 0.3674\end{array}\right], C_{3}=\left[\begin{array}{ll}1 & 0\end{array}\right]$.

The figure 2 shows the evolutions of the real and multimodel outputs in the validation phase, it illustrates the good accuracy of the elaborated base.

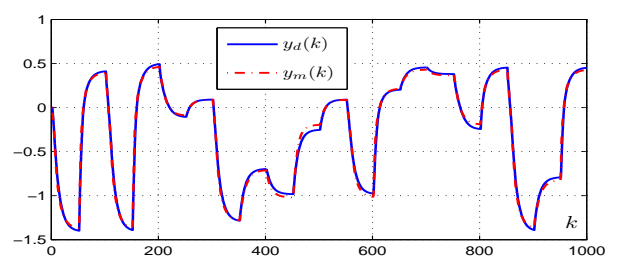

Fig. 2. Evolutions of the real and multimodel outputs.

In order to validate the identified model, two performance indices can be defined [26]. The first index is the Mean Square Error (MSE), its expression is given by:

$$
M S E=\frac{1}{N_{H}} \sum_{k=1}^{N_{H}}\left(y_{m}(k)-y(k)\right)^{2}
$$

with:

$y_{m}(k)$ is the multimodel output.

$y(k)$ is the system output.

The second index is the Variance Accounted For:

$$
V A F=\max \left\{1-\frac{\operatorname{var}\left(y^{N}-y_{m}^{N}\right)}{\operatorname{var}\left(y_{m}^{N}\right)}, 0\right\} \times 100 \%
$$

var denotes the signal variance.

The good adequation between the output of the nonlinear system and that of the multimodel can be clearly illustrated through the following values:

$$
M S E=0.0012, V A F=99.6727 \%
$$

The system output is available for measure after a time-delay. This dead-time occurs because of the time of transmission between the system and the control unit, communication system with low speed or sensor technology.

This time-varying delay is expressed in multiple integer of the sampling period and has the following expression:

$$
d(k)=3+2 \sin (0.015 k)
$$

The control strategy is applied to the system 31 with delayed output and described by decoupled multimodel. Firstly, the time-delay is not considered in the multi-observer synthesis as well as in the elaboration of the control law. 
The following figures illustrate the effect of the non consideration of the time-delay on the state estimation quality and subsequently on the control law.

Figure 3 illustrates the evolutions of the real and desired outputs as well as the control signal.
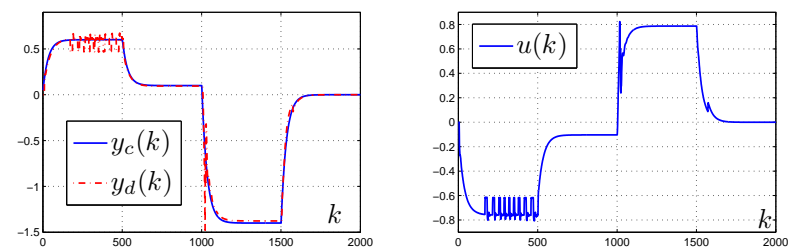

Fig. 3. Evolutions of the real and desired outputs (left). Evolution of the control signal (right) (non-consideration of time-delay).

The evolutions of the estimation errors of the partial states are given by the figures 45 and 6
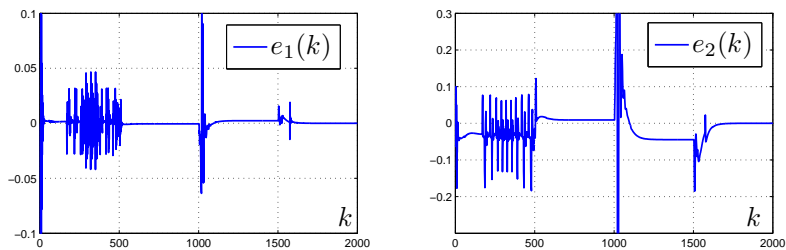

Fig. 4. Evolution of the estimation error of states 1 and 2 (non-consideration of time-delay).
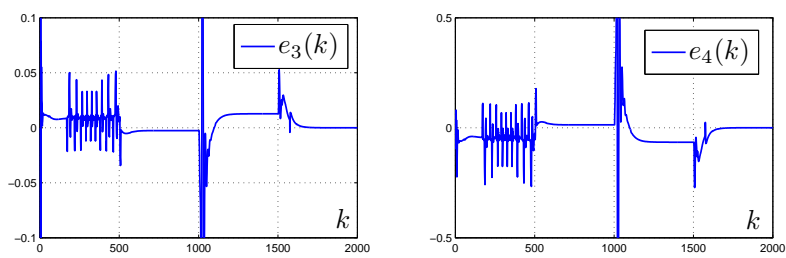

Fig. 5. Evolution of the estimation error of state 3 and 4 (non-consideration of time-delay).
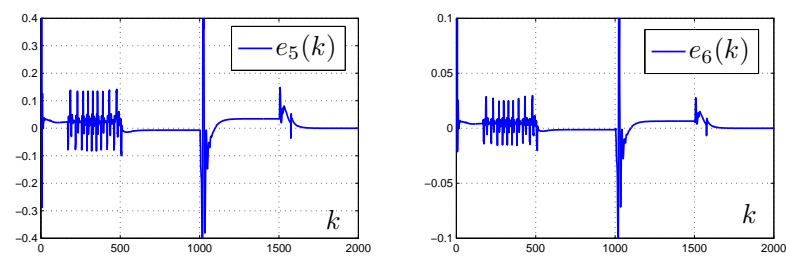

Fig. 6. Evolution of the estimation error of state 5 and 6 (non-consideration of time-delay).

The performance of the decoupled multimodel predictive control is considerably deteriorated in the case of non consideration of the time-delay in the observer synthesis and the control law elaboration.

Hereafter, the control law is synthesized taking into account the presence of the time-delay.

Figure 7 illustrates the evolutions of the real and desired outputs as well as the control signal.
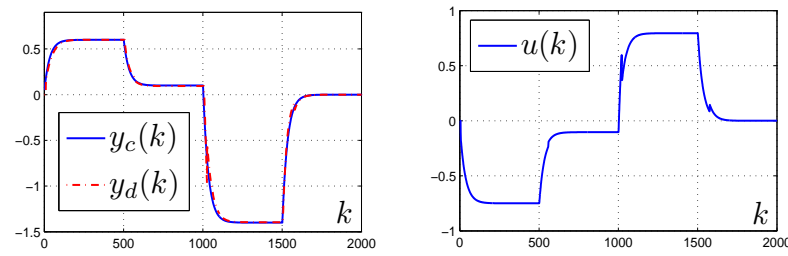

Fig. 7. Evolutions of the real and desired outputs (left). Evolution of the control signal (right) (consideration of time-delay).

The figure 8 shows the commutation between the partial controllers.

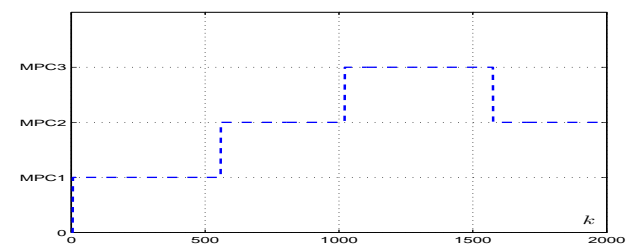

Fig. 8. Commutation between the local controllers (consideration of time-delay).

The evolutions of the real and estimated states as well as the estimation errors are shown in the figures 9,10 and 11
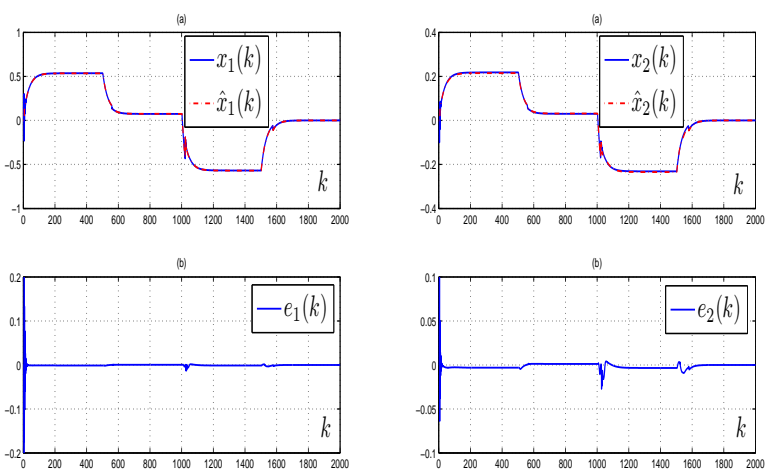

Fig. 9. (a) Evolutions of the state 1 and 2 and their estimates (b) Evolution of the estimation error (consideration of time-delay). 

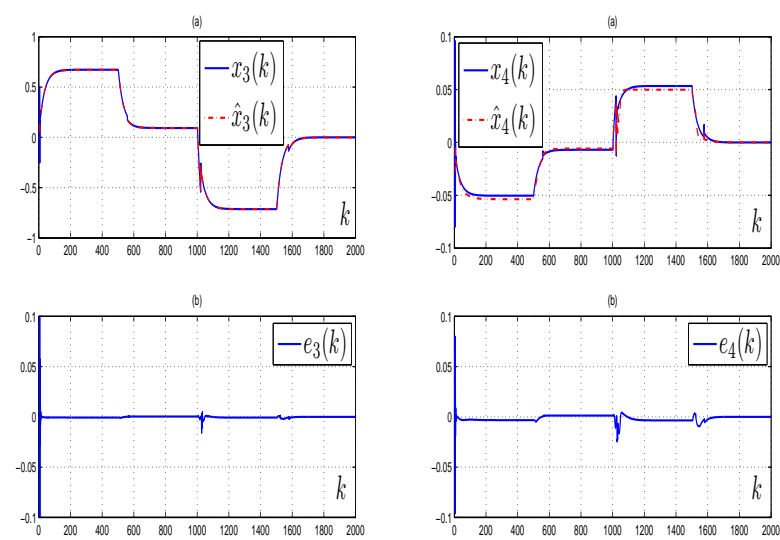

Fig. 10. (a) Evolutions of the state 3 and 4 and their estimates (b) Evolution of the estimation error (consideration of time-delay).
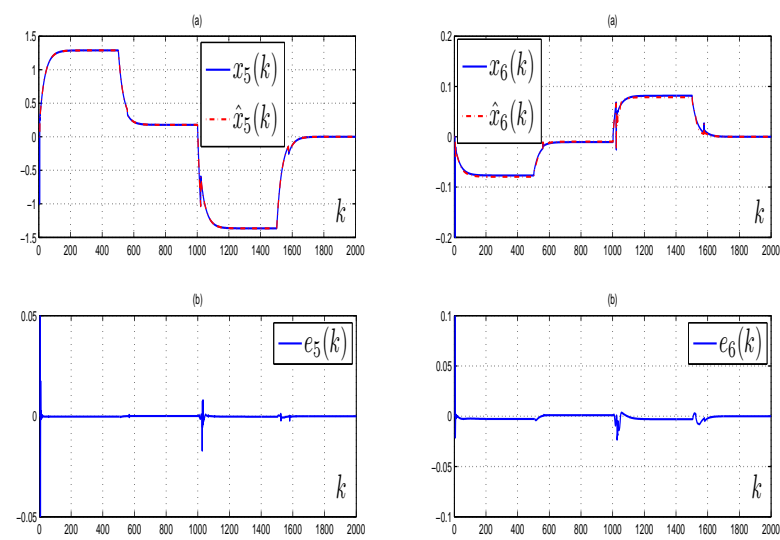

Fig. 11. (a) Evolutions of the state 5 and 6 and their estimates (b) Evolution of the estimation error (consideration of time-delay).

These figures show clearly that the consideration of a time-varying delay in the multi-observer design leads to a significant improvement in state estimation compared to the case of non consideration of time-delay in the multi-observer synthesis. This leads to an efficient decoupled multimodel predictive control that provides a good closed-loop performances compared with the case where the time-delay is ignored in the multi-observer synthesis.

\section{CONCLUSION}

In this paper, a multi-observer is synthesized for the state estimation of nonlinear systems with time-varying delay on the output measurements described by discrete-time decoupled state multimodel. These estimates are considered thereafter in the design of the decoupled multimodel predictive control. A supervisor enables the choice of the suitable partial controller that satisfies the closed-loop objectives through a performance criterion. The significance of this control strategy has been illustrated by simulation results that show good closed-loop performances. In our future works, a multiobserver will be investigated for the state estimation of discrete-time uncertain nonlinear systems with delayed output measurements.

\section{REFERENCES}

[1] J. P. Richard. "time-delay systems: an overview of some recent advances and open problems". Automatica, 39(10):1667-1694, 2003.

[2] V. Manikonda, P.O. Arambel, M. Gopinathan, R.K. Mehra, and F.Y. Hadaegh. "a model predictive control-based approach for spacecraft formation keeping and attitude control". In American Control Conference, pages 4258-4262, 1999.

[3] M. Tillerson, G. Inalhan, and J. P. How. "co-ordination and control of distributed spacecraft systems using convex optimization techniques". International Journal of Robust and Nonlinear Control, 12(2-3):207-242, 2002.

[4] WB Dunbar and RM Murray. "model predictive control of coordinated multi-vehicle formations". In Proceedings of the 41st IEEE Conference on Decision and Control, 2002.

[5] S. Domek. "switched state model predictive control of fractional-order nonlinear discrete-time systems". Asian journal of control, 15(3):658-668, 2013.

[6] F. Yan and P.H. Hu. "state feedback model predictive control to complex systems with multi-time delays ". Proceedings of the 5th World Congress on Intelligent Control and Automation. Hangzhou, China, pages 946-950, 2004.

[7] X.B. Hu and W.H. Chen. "model predictive control for constrained systems with uncertain state-delays". International Journal of Robust and Nonlinear Control, 14(17):1421-1432, 2004.

[8] H.N. Nounou and K.M. Passino. "fuzzy model predictive control: Techniques, stability issues, and examples". Proceedings of the IEEE International Symposium on Intelligent Control, pages 423-428, 1999.

[9] D. Matko, K. Kavsek-Biasizzo, I. Skrjanc, and G. Music. "generalized predictive control of a thermal plant using fuzzy model". In American Control Conference. Chicago, Illinois, pages 2053-2057, 2000.

[10] H. Sarimveis and G. Bafas. "fuzzy model predictive control of non-linear processes using genetic algorithms". Fuzzy Sets and Systems, 139(1):59-80, 2003.

[11] C.L. Su and S.Q. Wang. "robust model predictive control for discrete uncertain nonlinear systems with time-delay via fuzzy model". Journal of Zhejiang University SCIENCE A, 7(10):1723-1732, 2006

[12] A. Germani, C. Manes, and P. Pepe. "a new approach to state observation of nonlinear systems with delayed output". Automatic Control, 47(1):96-101, 2002.

[13] S. Ibrir, W. F. Xie, and C.-Y. Su. "observer design for discrete-time systems subject to time-delay nonlinearities". International Journal of Systems Science, 37:629-641, July 2006.

[14] N. Kazantzis and R. Wright. "nonlinear observer design in the presence of delayed output measurements". Systems and Control Letters, 54(9):877-886, 2005.

[15] T. A. Johanson and A. B. Foss. "nonlinear local model representation for adaptive systems". volume 2, pages 677-682, 1992. 
[16] K. Tanaka and M. Sano. "a robust stabilization problem of fuzzy control systems and its application to backing up control of a truck-trailer". IEEE Transactions on fuzzy systems, 2(2):119-134, 1994.

[17] A.M. Nagy Kiss. " Analyse et synthse de multimodles pour le diagnostic. Application une station d'puration". $\mathrm{PhD}$ thesis, Institut National Polytechnique de Lorraine, France, 2010.

[18] J. Abonyi, R. Babuska, and F. Szeifert. "modified gath-geva fuzzy clustering for identification of takagi-sugeno fuzzy models". IEEE Transactions on Systems, Man and Cybernetics, Part B : Cybernetics, 32(5):612-621, 2002.

[19] G. Mourot, K. Gasso, and J. Ragot. Modelling of ozone concentrations using a takagi-sugeno model. Control Engineering Practice, 7(6):707-715, 1999.

[20] Y. Batmani and H. Khaloozadeh. "on the design of observer for nonlinear time-delay systems". Asian Journal of Control, 16(5):1-11, 2013.

[21] Y.Y Cao and P.M. Frank. "analysis and synthesis of nonlinear time-delay systems via fuzzy control approach". Transactions on Fuzzy Systems, 8(2):200-211, 2000.

[22] Y.Y Cao and P.M. Frank. "stability analysis and synthesis of nonlinear time delay systems via takagi-sugeno fuzzy models". Fuzzy Sets and Systems, 124:213 - 229, 2001.

[23] H. Gassara, A. El Hajjaji, and M. Chaabane. "commande tolrante aux dfauts actionneurs base sur un observateur adaptatif pour les modles flous t-s retard variable dans le temps born. In Journes Doctorales MACS, JDMACS'2011, Marseille (France), 2011.

[24] C. Lin, Q.G. Wang, and T.H.Lee. " LMI approach to analysis and control of Takagi-Sugeno fuzzy systems with time-delay". Springer-Verlag, 2007.

[25] C. Latrach, M. Kchaou, A. El Hajjaji, and M. Chaabane. "robust stabilization conditions for time-delay fuzzy systems using fuzzy weighting-dependent approach". In 19th Mediterranean Conference on Control and Automation, pages 20-23, 2011.

[26] R. Orjuela. "Contribution l'estimation d'tat et au diagnostic des systmes reprsents par des multimodles". $\mathrm{PhD}$ thesis, Institut National Polytechnique de Lorraine, France, 2008.

[27] R. Orjuela, B. Marx, D. Maquin, and J. Ragot. State estimation of nonlinear discrete-time systems based on the decoupled multiple model approach. In 4th International Conference on Informatics in Control, Automation and Robotics, ICINCO 2007, Angers, France, 2007.

[28] R. Orjuela, B. Marx, D. Maquin, and J. Ragot. "a decoupled multiple model approach for state estimation of nonlinear systems subject to delayed measurements ". In In $3 r d$ IFAC Advanced Fuzzy and Neural Network Workshop, AFNC, Valenciennes, France, 2007.

[29] J. Prakash and R. Senthil. "design of observer based nonlinear model predictive controller for a continuous stirred tank reactor". Journal of Process Control, 18:504-514, 2008.

[30] J.H. Lee and N.L. Ricker. "extended kalman filter based nonlinear model predictive control". Industrial Engineering and Chemistry Research, 33:1530-1541, 1994.

[31] S. Dan. "optimal state estimation-kalman h inf and nonlinear approaches". A John Wiley \&Sons, Inc., Publication, 2006.

[32] M. Geetha, J. Jerome, and V. Devatha. "design of state estimation based model predictive controller for a two tank interacting system". International Conference on Design and Manufacturing (IConDM2013), 64:244-253, 2013.

[33] A. Messaoud, M. Ltaief, and R. Ben Abdennour. "supervision based a multipredictor for an uncoupled state multimodel predictive control". In The 6th International conference on electrcal systems and automatic control, JTEA'2010, Hammamet, Tunisia, 2010.

[34] A. Messaoud, M. Ltaief, and R. Ben Abdennour. "supervision based on partial predictors for a multimodel generalized predictive control: experimental validation on a semi-batch reactor". International Journal of Modeling, Identification and Control, 6(4):333-340, 2009.

[35] O. Pags, C. Bernard, O. Raul, and M. Pascal. "control system design by using a multi-controller approach with a real-time experimentation for a robot wrist". International Journal of Control, 75(16):1321-1334, 2002.

[36] R. Orjuela, D. Maquin, and J. Ragot. "nonlinear system identification using uncoupled state multiple-model approach". In 4th Workshop on Advanced Control and Diagnosis, ACD'20-06, Nancy, France, 2006.

[37] A. Savran. "discrete state space modeling and control of nonlinear unknown systems". ISA Transactions, 52:795-806, 2013. 